\title{
Research on Emotional Marketing and Copywriting of NetEase Cloud Music
}

\author{
Yasi Huang ${ }^{1, *}, \dagger$, Yazhao Huang ${ }^{2, *},{ }^{*}$ and Pengcheng $\mathrm{Li}^{3, *}, \dagger$ \\ ${ }^{1}$ Tianjin Experimental High School, 300074, Tianjin, China \\ ${ }^{2}$ Tianjin Experimental High School, 300074, Tianjin, China \\ ${ }^{3}$ Beijing 101 high school, 100091, Beijing, China \\ *Corresponding author. Email: guanghua.ren@gecacdemy.cn \\ These authors contributed equally.
}

\begin{abstract}
Nowadays, NetEase cloud music as a new platform has appeared in our views field for 8 years. On the one hand, NetEase Cloud Music has succeeded in attracting a large audience and users by buying many exclusive rights and shifting its focus to independent musicians and smaller offline music venues. From another perspective, NetEase Cloud Music has achieved great success in publicity and advertising by arousing the resonance of users through emotional marketing of copywriting. This paper will analyse the emotional marketing strategy and existing problems of NetEase Cloud Music and provide solutions to the problem.
\end{abstract}

Keyword: NetEase cloud music, Emotional marketing, Copywriting, Strategies.

\section{INTRODUCTION}

Nowadays, the competition of internet music is more and more fierce. Since it launched in 2013, NetEase cloud music has received critical acclaim, and in 2017, The new user retention rate of NetEase Cloud Music is number one among music apps [1]. It surpasses many other famous music apps like QQ music. China Mobile Internet Industry Development Analysis report in the first quarter of 2019 shows in the mobile music market, NetEase Cloud Music's user engagement ranked first, Leading the industry with 33.5 percent [2]. In the mobile music market, the user viscosity of this app maintains a high ranking. Such great success cannot be separated from its excellent emotional marketing strategy and characteristic copywriting.

$\mathrm{Mu}$ proposed that emotional marketing could win more users for NetEase cloud music. It takes advantage of the similarity of users' emotions to connect the user with the user to form a bond: product-users-more users and create value together. The app is launching a music community, so people could chat and share their favourite music with friends who have the same taste in music. In this circle, people could update at any time. The app is also providing different sharing platforms to satisfy young people's and other people's needs. NetEase cloud music has a Characteristic playlist and accurate delivery functions to adapt to the need of people [3]. Li thought that emotional marketing focuses on the personal feelings of consumers. Through the improvement of product functions, products and consumers have a kind of emotional communication. By this communication, NetEase cloud music wins the preference of consumers. A great example was in 2017, and its Music Annual Report almost took over the entire circle of friends. The music app used users' data recordings from user listening reports and summarized the year's keyword. This report summarized the hours you heard the whole year, make a list of your favourite songs and display the number of times you listened to each song, and summarize your favourite singer of the year. Not only these, but it also designed pretty background and chose suitable background music. This music report used this approach to successfully establish an emotional connection with regular users and let them stay and attract new users at the same time [4]. Wang found that NetEase cloud music uses advertisements in public to do emotional marketing. For example, 85 music reviews collected from 4 billion reviews were painted with white characters on a red background in the car of Hangzhou subway line one and Hangzhou Jiangling Rode subway station. Plenty of people took photos with their favourite music reviews and thought highly of it. According to the final data, NetEase cloud music has gained wide attention and discussion, the rankings on the charts had also improved 
dramatically. Wang thought that success comes from the team of NetEase cloud music that has deep insight into people's wishes. Young people in a big city often fall confused in their busy work and life. These moving music reviews resonate with people emotionally and successfully capture their attention. By doing this, NetEase cloud music attracted lots of public attention and have more users [5].

According to our research, lots of scholars research the emotional marketing of NetEase cloud music. Most of them focus on the special and convenient functions they have an effective means of publicity. Few scholars research copywriting's marketing strategy, so we will focus on this to do the research in this paper.

The mean research methods of this paper are literature analysis, case study, and summarization and introduction. The main research ideas of this paper follow four parts. First, NetEase cloud music's development and platform introduction. Second, analysis of emotional marketing and copywriting of NetEase cloud music. Third, analysis of the problem existing in emotional marketing strategy. Fourth, optimization of this strategy.

Our excepted result is to analyze NetEase cloud music's advantage and learn something from it to promote the development of China's digital media music platform.

\section{METHOD}

\subsection{Literature analysis method}

Literature analysis is a method to obtain data by investigating literature according to certain research purposes or topics to understand the research comprehensively and correctly [6]. A great deal of literature data is necessary to know more about emotional marketing strategy and paperwork's influence on NetEase music. Through different opinions and different aspects, people can fully understand the topic, which is beneficial for people to support viewpoint easier. This article will use this method and relevant data to analyse NetEase music in different periods. To show how emotional marketing strategy and paperwork in NetEase music develop and why it can attract so many people in such a competitive situation.

\subsection{Case Study}

The case study is a kind of empirical study. It means studying phenomena that are happening without getting divorced from real life [7]. By analysing classic cases, people could know how NetEase music translates its emotional marketing strategy into effective action to appeal to many consumers. Also, according to the case of success or failure, it is a good chance for NetEase music to know what and how their consumers thought and felt about their work. So that they could adjust their strategy to meet customer needs, we would like to show several typical cases in this article and analyse the importance of NetEase music's emotional marketing strategy and its paperwork. In this way, we could understand how the strategy and paperwork take effect.

\subsection{Summarization and induction}

Summarization and induction is a method that means the reasoning deriving general knowledge from individual knowledge. It derives a possible true conclusion from known true premises [8]. According to the analysis about emotional marketing strategy in NetEase music and its paperwork, summarizing and concluding both of the advantages and the drawbacks. That could help NetEase music find potential problems and put forward the corresponding countermeasure in time. To promote music platforms to operate healthily. Therefore, we will collate the data and the case we fund, analysing the advantages and disadvantages of NetEase's strategy and paperwork. To show how they can keep their merit, what could lead to a mistake, and how they could prevent it.

\section{RESULT}

\subsection{Development Overview and Platform Profile of NetEase Cloud Music}

NetEase Cloud Music is a music product developed by NetEase, which is the achievement of NetEase Hangzhou Research Institute. Relying on professional musicians, DJ, friend recommendation, and social functions, online music services focus on the song list, social, big-name recommendation, and music fingerprint, with a song list, DJ program, social and geographical location as the core elements, focusing on discovery and sharing. On April 23rd, 2013, NetEase released the latest product and application-NetEase Cloud Music, and since its release, its application has expanded a variety of products. By including but not limited to supporting independent musicians to create works, striving for copyright to expand the music library, NetEase Cloud Music conducts accurately pushing to grasp the needs of users and improving the utilization rate of the music library. First of all, independent musicians do not cooperate with any record companies. By supporting independent musicians, such as Zhao Lei, Xie Chunhua, and fandongdemao, they can produce a series of excellent independent music works; through a large number of works from independent musicians, NetEase Cloud Music can effectively control copyright from the source, expand music library and enrich music content. Secondly, besides expanding the music library by supporting independent musicians, NetEase Cloud Music also strives for the copyright of songs. It enhances its copyright hard power based on sublicense cooperation with other platforms. NetEase Cloud Music has reached 
an exclusive copyright strategic cooperation with Avex, the largest entertainment group in Japan, and reached strategic cooperation with $\mathrm{KKBOX}$, the top digital music service brand in Asia. These measures show that NetEase Cloud Music is actively striving for copyright, expanding the music library, and enriching the content of music products. Moreover, NetEase Cloud Music can provide personalized, customized services for users according to their preferences and needs based on big data, aiming at attracting customers and popularity from a wide audience [9].

\subsection{Analysis of the current situation of the emotional marketing strategy of NetEase Cloud Music and its copywriting}

\subsubsection{Analysis of the current situation of the emotional marketing strategy of NetEase Cloud Music copywriting}

NetEase Cloud Music has launched emotional products that meet the needs of minority music lovers. NetEase Cloud Copywriting has been spread among the broad audience with the concepts of touching and empathy and has formed a wide communication network quickly. Since its launch in April 2014, NetEase Cloud Music has become the largest mobile music community in China in one fell swoop. Besides its products can produce a lot of high-quality content, it also shows its creativity in brand marketing communication. In the aspect of brand emotional marketing communication, AISAS mode has the most suitable communication effect, which is more suitable for the background of the Internet and mobile applications and more suitable for the changes of consumers' roles and psychology in the new media environment. A: Attention (brand releases emotional information to awaken emotional psychology of consumers), I: Interest (stimulating emotional interest, such as excitement, loneliness, happiness), S: Search (consumers actively search for brand emotional products, search for other consumers' purchase experiences, emotional comments, etc.) is classified as emotional marketing propaganda channels. A: Action (conforming to the expected emotional identity of consumers and promoting purchase action) is set as an emotional product analysis for households to consume, and S: Share (consumers have a good emotional experience and actively share their emotional experience to tagged communities and emotional, cultural circles) is set as a way for users to share their emotions.

\subsubsection{Analysis of the current situation of the emotional marketing strategy of NetEase Cloud Music advertisement}

The ultimate goal of advertising is to persuade consumers to buy, and Emotional advertising caters to the specific emotional needs of consumers. In the form of symbolism and other arts, the brand is implicitly publicized. The specific emotional color in emotional advertisement can quickly open the hearts of consumers, deeply impress consumers. At the same time, it can skillfully publicize brand information, which makes consumers have further consumption behavior after psychological changes. Emotional advertising promotes products imperceptibly by marketing consumers' emotions, and consumers can generate corresponding interest and trust in products with emotional resonance [10]

\subsection{Analysis of the Problems Existing in NetEase Cloud Music Emotional Marketing Strategy}

\subsubsection{Occupying network resources}

Some copywriting will cause heated discussion and debate among people with different views, occupying network resources and popularity. On the one hand, occupying public resources with unrealistic content such as copywriting is unfavourable to the overall network environment. On the other hand, the fierce discussion on the network is indispensable for NetEase Cloud Music to accumulate users and popularity, which is extremely contradictory.

\subsubsection{Timeliness of information}

The popularity and freshness of copywriting will deteriorate after a certain period. For example, the same copywriting appears countless times in Zhihu or friends circle, which will lead to aesthetic fatigue and obsolescence for users, resulting in a large number of users switching to other software and losing users.

\subsubsection{The number of copyrights}

It is difficult to attract more consumers and audiences to join the large group of NetEase Cloud Music by emotional marketing alone. The reason is that other music companies occupy too many exclusive music rights, which further reduces the market of NetEase Cloud Music. On the contrary, the number of songs or singer Copyrights of NetEase Cloud Music is far less than other music platforms due to the lack of capital and the late entry into the market

\section{DISCUSSION}

\subsection{Change the direction of copywriting.}

Although copywriting is very literary, its words are elegant, and such copywriting does not have practical value. These cannot let people think about something or learn something from it. Many people resent the fact that 
flashy copywriting occupies a lot of public resources. So NetEase cloud music could focus on some valuable topics when they are preparing copywriting, like the social problem. Now more and more musicians are willing to write songs that reflect social reality. Some songs are about equality between men and women, and some songs stand up to bullying. Some songs describe people of different occupations who have difficulties in life and work. NetEase cloud music could write some views on these social issues and enlighten them, or encourage more people to pay attention to these issues and care even help some different groups in society. In this way, copywriting has valuable content. On this basis, NetEase cloud music could embellish the copywriting with beautiful language as they did before. This kind of copy can make people feel fresh and think about social life at the same time. Such copywriting is sure to provoke heated debate. By doing this, The atmosphere of discussion in society will become more positive and meaningful, and NetEase cloud music could get more heat and leave a good impression on consumers at the same time.

\subsection{Meet personal emotional differences and needs}

Good copywriting can really impress users, but its freshness will wear off over time, then it will lose users' goodwill and lead to the leaving of users. This is an inevitable result of copywriting because the freshness of gorgeous language can't last long. If NetEase cloud music wants to retain customers for a long time, it could keep improving on emotional marketing, which is the app's advantage. Use an emotional connection with consumers to retain them. An important part of it is to identify each user's emotional needs and try to meet them. NetEase cloud music could use questionnaires or big data to collect consumers' experiences and evaluations. Using this data to make personalized adjustments and recommendations for individual consumers. Satisfying customers in as much detail as possible, like the design of cover and background, recommended playlist, and some other detail design. This gives the user a sense that they are understood and valued. Users will feel more positive about the software and be willing to use this app for a long time. Only by taking consumers as the main body, paying attention to the emotional experience of different users, and integrating the experience into products to meet the needs of consumers, can consumers be satisfied, and the product becomes more competitive.

\subsection{Make good emotional public relations}

Public relations are becoming increasingly important in marketing. Software featuring emotional marketing such as NetEase cloud music should pay more attention to emotional public relations. NetEase cloud music needs to find ways to strengthen the emotional communication with customers through questionnaires and other forms of consumer participation in the marketing activities so that consumers could have a deeper understanding of the company and products, and consumer goodwill and trust in the product will rise.

The first competition is the point of sale, and then the second competition is after-sales service. Through a competitive and attractive commitment, enterprises persuade the purchase and build the brand image by fulfilling the promise with both quality and quantity. NetEase cloud music could also increase customer loyalty in this way, to differentiate itself from other competing companies in service. The emotional service make consumers trust this product and achieve a balanced and harmonious relationship between the product and the customer. This way can retain the original consumers and attract more consumers, and use this trust to stabilize customers for the long term and prevent customers from leaving because of the stale copywriting.

\subsection{Improve software quality}

Emotional marketing in product marketing plays the role of icing on the cake. It puts the cart before the horse to make up for the lack of product quality with good emotional marketing. Continuous improvement of software quality is essential for long-term product development. NetEase cloud music needs to timely correct some technical problems and according to users' needs to continuously optimize and upgrade the product.

When it comes to music software, consumers naturally prefer apps that have a wider range of music and songs they like. NetEase cloud music urgently needs to buy more music copyright to expand its library, especially some classic songs and hit songs. These kinds of songs are loved and noticed by plenty of people. If this app has the copyrights to these songs, it can attract a lot of fans and add beautiful copywriting and emotional marketing strategies. It will have a competitive advantage over other music apps and is sure to attract more consumers.

\section{CONCLUSION}

In conclusion, NetEase music as a burgeoning music application appear in our visual fields recently. Not only having a solid foundation of technology and some exclusive copyrights but also based on the special distribution ways to attract users such as using emotional marketing strategy in the advertisement of the application. In other words, special advertisements can elicit emotions and reactions in wide audiences and users. The application is based on the AISAS system to distribute the content of the music. It is more suitable for the context of the Internet and mobile applications and more in line with the changes in consumers' roles and 
psychology in the new media environment. However, there are some difficult problems waiting to be solved. For instance, some copywriting will cause conflicts between audiences, occupy network resources and cause unnecessary wasting of resources. Moreover, timeliness is an indispensable part of all of the messages. For consumers, the same advertisement and copywriting will cause aesthetic fatigue. Lastly, Due to the lack of capital and the late entry into the market, the number of songs' or singers' copyrights of NetEase Cloud Music is far less than that of other music platforms. However, the author gives some advice to improve the situation and push the application incessantly. First of all, changing the direction of copywriting and paying attention to the social hot spots and current news. NetEase music can focus on some valuable topics when the platform preparing advertisements and copywrites. Secondly, meeting personal differences and needs. Thirdly, making a flexible emotional public relation is also an indispensable and important part of the development of NetEase cloud music. Through several ways, NetEase music can improve the situation and become better.

\section{REFERENCES}

[1] https://sohu.com/a/130168716_570240

[2] DuoJiao Fu. Research on emotional marketing of NetEase Cloud Music, Shenyang Normal University, 2020.

[3] MengSha Mu. Research on emotional marketing Strategy of NetEase Cloud Music, Rural economy and science and technology, 2019, 30(22): 125-126.

[4] LuYi Li Research on emotional marketing Strategy of NetEase Cloud Music, Media BBS, 2019, 2(06): 170-171.

[5] ZhuJun Wang. NetEase Cloud Music: Delve into users' emotional marketing, International public relations, 2017(05):74-75. DOI:10.16645/j.cnki.cn11-5281/c.2017.05.029

[6]https://baike.baidu.com/item/\%E6\%96\%87\%E6\%A1 $\% 88 \%$ E8\%B0\%83\%E6\%9F\%A5\%Е6\%B3\%95/59 14547

[7]https://baike.baidu.com/item/\%E6\%A1\%88\%E4\%B Е\%8B\%Е5\%88\%86\%Е6\%9E\%90\%Е6\%B3\%95/4 451542

[8] http://mip.wenshubang.com/shenghuo/239590.html

[9] Xie Ting. Product Strategy Analysis of NetEase Cloud Music under the Trend of IP Industrialization. Modern Marketing (Business Edition), 2021 (05): 75-77.

[10] Wang Jingyao. Research on brand emotional marketing communication strategy under online media environment, Guizhou Minzu University, 2020 . 